\title{
Defensive burying in the rat: A behavioral field analysis
}

\author{
EDWARD J. PEACOCK and PAUL T. P. WONG \\ Trent University, Peterborough, Ontario K9J 7B8, Canada
}

\begin{abstract}
Time spent in various behaviors by the rat was recorded in a defensive burying paradigm. Experiment 1 revealed that rats spent more time burying the shock prod than a control prod and that doubling the size of the test chamber did not have a significant effect on the time spent in any behavior. In Experiment 2, the location of bedding material in a two-compartment test chamber was found to affect the occurrence of burying (both the shock and control prods) and burrowing behavior. Burying did not occur when bedding was not available in the shock compartment but was located in the escape compartment. Burrowing was more likely to occur when bedding was in both compartments than when it was in only one compartment. Immobility and escape latencies were shorter than burying latencies in all subjects. Burying was viewed as belonging to a second stage of defensive behavior.
\end{abstract}

A new paradigm for the study of aversively motivated behavior has received considerable attention recently. Pinel and Treit (1978) found that rats shocked by one of two identical prods mounted on opposite walls of a test chamber returned to the shock prod and buried it using material available in the test chamber. Further investigation has indicated that the phenomenon, referred to as defensive burying, will occur over a variety of shock-test intervals (Pinel \& Treit, 1978), with a variety of available materials (Pinel \& Treit, 1979), and following different sources of aversive stimulation (Terlecki, Pinel, \& Treit, 1979; Wilkie, MacLennan, \& Pinel, 1979). Thus, defensive burying appears to be a robust and reliable phenomenon.

Pinel and Treit $(1978,1979)$ argue that Bolles's (1970) hypothesis of species-specific defense reactions cannot readily account for defensive burying, which involves approach to the source of stimulation. According to Bolles (1970), whenever an aversive event occurs, an animal's behavioral repertoire is immediately restricted for a period of time to a narrow class of behavior. The animal will take flight, freeze, or threaten aggression. Although Pinel and Treit (1978, 1979) maintained that the occurrence of freezing, fleeing, and aggressive behavior was rare in their studies, they have never reported systematic measures of any behavior other than burying. Since the mean time spent burying typically represents only a fraction of the total observation time in these studies, it is not

This study was partially supported by a grant from the Natural Sciences and Engineering Research Council of Canada to the second author. The authors gratefully acknowledge the assistance of Jean Peel in analyzing the videotapes. Portions of this paper were presented at the annual meeting of the Canadian Psychological Association in Toronto, June 1981. Requests for reprints should be addressed to P. T. P. Wong, Department of Psychology, Trent University, Peterborough, Ontario K9J 7B8, Canada. clear what other behaviors occur in this situation.

The relationship between defensive burying and other defense reactions is also brought into question by the results of a study by Pinel, Treit, Ladak, and MacLennan (1980), which indicated that the amount of burying appears to be influenced by the opportunity to perform competing defensive responses. It was found that increasing the opportunity for flight by increasing the size of the test chamber or by providing an escape compartment reduced but did not eliminate defensive burying.

A striking feature of defensive burying reported by Pinel and Treit (1978) is the consistency with which rats selectively buried the shock prod. In one experiment, every rat shocked by one of two identical prods mounted on opposite walls of the test chamber buried only the prod through which it was shocked. In our initial attempts to demonstrate defensive burying using a similar procedure, we not only found burying to be less consistent, but also observed freezing, digging, and running away from the shock prod. Therefore, it seems desirable to gain a more complete account of defensive burying by examining this phenomenon within the context of other behaviors occurring in the test situation. The present two experiments attempted to accomplish this by applying a behavioral field approach (Wong, 1977, 1979) to the study of defensive burying.

\section{EXPERIMENT 1}

The purpose of this experiment was to examine the behavioral context in which defensive burying occurs. The effect of varying the size of the test chamber was also investigated. It was expected that increasing the size of the test chamber would decrease the amount of time spent burying. On the basis of previous re- 
search (Wong, 1979) and our preliminary observations of defensive burying, seven mutually exclusive categories of behavior were identified. Exploration consisted of all behaviors that involved general activity by the rat (i.e., locomotion, rearing, sniffing, hopping). Grooming included all activities directed toward various parts of the body (i.e., face washing, scratching, tail biting, and licking of the body). Immobility consisted of crouching, lying, sitting, or standing still on at least three feet, with the body completely motionless except for small and slow lateral movements of the head. Chewing consisted of bringing the teeth in contact with any material other than a body part. Digging involved repeated movement of the snout and paws in a manner directed at pulling bedding material toward and under the body. Burying the shock prod consisted of movement of the front paws and snout in a pushing manner so as to move material (either forward or sideways) toward the shock prod. Similarly, burying the control prod consisted of the same behavior as burying the shock prod except that it was directed at the control prod.

\section{Method}

Subjects. The subjects were 20 naive male adult rats of the LongEvans strain that were bred and reared in the animal colony at Trent University. The animals were housed individually in wirebottom cages in a room with a 16:8-h light/dark cycle. Purina rat chow pellets and water were available ad lib in home cages.

Apparatus. The testing apparatus consisted of a Plexiglas chamber $44 \times 60 \times 44 \mathrm{~cm}$. A removable Plexiglas partition could be placed so as to divide the chamber into a single-size compartment the same size as the Pinel and Treit (1978) apparatus $(44 \times 30 \times$ $44 \mathrm{~cm}$ ). With the partition removed, the chamber could be used as a double-size compartment. The floor of the chamber was covered with $5 \mathrm{~cm}$ of ordinary wood shavings to serve as bedding material. Two removable Plexiglas prods, each measuring $.6 \times .7 \times 6.3 \mathrm{~cm}$ and wrapped with two uninsulated wires, were fastened to the center of the walls at opposite ends of one of the single compartments $2 \mathrm{~cm}$ above the level of the bedding material. One prod (arbitrarily predetermined) served as the shock prod, while the other served as a control prod.

Shozk generated by a BRS Shock Generator-Scrambler Model SGS 003 set at $8 \mathrm{~mA}$ was delivered to the two wires of the shock prod. A Sony Model A V3400 video camera and recorder were used to videotape each rat's behavior during the test session. Behavioral analysis of the videotapes was accomplished by operating a manual keyboard that activated time recorders.

Procedure. The 20 animals were divided randomly into two groups. One group became the single-size condition, being exposed and tested in the single-size compartment, while the other group became the double-size condition, being habituated and tested in the double-size compartment. Handling and habituating to the appropriate test compartment were done in squads of three to four for 30-min periods on 4 consecutive days. On Day 5 , the two prods were fixed in position and rats were placed individually in the center of the appropriate test compartment. Each subject was allowed time to touch each prod with a forepaw at least once prior to the introduction of shock. Then, when the rat again touched the shock prod with a forepaw, the experimenter initiated the shock, which terminated with the withdrawal of the paw from the shock prod. The animal's behavior was videotaped for $15 \mathrm{~min}$ following the shock. At the end of this session, the height of piles of bedding within $10 \mathrm{~cm}$ of the prods was measured.
The interrater reliability of the seven behavioral categories was established by having two observers independently score 10 of the videotape sessions. The correlation coefficients between the two sets of scores ranged from .88 (burying the shock prod) to .99 (grooming).

In this and the next experiment, logarithmic transformations of the time data were performed to normalize the distribution. Analyses of variance were performed on the transformed data; however, the means reported for both studies are based on actual time scores.

\section{Results and Discussion}

Size of the test chamber did not significantly affect the time spent in any of the seven observed behaviors in a comparison of the single-size condition with the double-size condition (all ps $>.05$ ). There was also no significant difference in either situation in the time spent burying the shock prod and the control prod. Therefore, the results from these two conditions were combined for further analyses. Our results were contrary to the expectation that increasing the size of the test chamber would decrease the amount of burying. The chamber-size effect reported by Pinel et al. (1980) might be due to the fact that they used a single-prod paradigm and a wider range of test-chamber sizes.

Considering all subjects and collapsing across conditions, the mean times spent in exploration, immobility, chewing, grooming, burying the shock prod, burying the control prod, and digging were 561.5, $183.1,50.7,48.4,34.9,15.5$, and $10.1 \mathrm{sec}$, respectively. Significantly more time was spent burying the shock prod than burying the control prod $[F(1,19)=4.90$, $\mathrm{p}<.05]$. There was, however, no significant difference $(p>.05)$ in the height of the bedding at the shock prod (mean $=8.1 \mathrm{~cm}$ ) and the height at the control $\operatorname{prod}($ mean $=6.9 \mathrm{~cm})$.

Burying behavior was found to be neither very consistent nor clearly directed. Even though significantly more time was spent burying the shock prod, animals also buried the control prod. Three of the 20 animals did not exhibit any burying at all. For the remaining subjects, only one animal did not bury the control prod. Five animals spent more time burying the control prod than the shock prod. These results contrast with those presented by Pinel and Treit (1978), who reported that animals selectively buried only the shock prod.

Although the height of the piles of bedding at each respective prod significantly correlated with the amount of time spent burying the shock prod $(r=.76)$ and the control prod $(r=.85)$, this measurement seems open to considerable error. Animals frequently disturbed the piles by behaviors subsequent to burying, which may explain why there was no significant difference in the height of the piles at the two prods even though significantly more time was spent burying the shock prod. Furthermore, time spent in burying appeared to be a more useful measure because it could be directly related to time measures for other behaviors. 
Therefore, time spent in burying is recommended for future studies of defensive burying.

\section{EXPERIMENT 2}

Although there was no measure of the latency to burying behavior in Experiment 1, observation indicated that burying behavior did not appear to be an immediate defensive response. Rather, it emerged after several minutes. We also observed burying-like behavior that was not directed at either prod; this behavior consisted of rooting, pushing, and spraying of bedding material (e.g., toward a corner). This buryinglike behavior was arbitrarily classified either as being directed toward one of the prods or as exploration, although burrowing seems to be a more accurate description of such behavior.

Burrowing has been found to be a species-specific behavior important for the survival of rats in a natural environment (Boice, 1977). To construct a burrow, rats must displace material in forward, sideways, and backward directions using paws and snout. Therefore, in this experiment, the category of burrowing was used to include digging, pushing, rooting, or spraying of the bedding that was not directed at the prods. This new behavioral category, which incorporates digging and undirected burying-like behavior not specifically aimed at the prods, represents an important aspect of the behavioral field in the defensiveburying paradigm. Burying, while sharing some behavioral characteristics with burrowing, is nevertheless distinguishable by the fact that it is directed at an identifiable object (i.e., the prod).

The purpose of Experiment 2 was to further investigate defensive burying in a double-compartment test chamber that permitted animals to escape following shock. The availability of bedding material was manipulated by locating bedding in only the prod side, in only the escape side, or in both sides. We also recorded the latency of occurrence of each category of behavior and the latency to escape (entry into the escape compartment).

On the basis of Bolles's (1970) hypothesis, it was predicted that latencies to immobility and escape would be shorter than burying latencies. It was also expected that burying would be affected by the location of the bedding, while burrowing would be affected by the total area of bedding. In other words, it was expected that rats would not bury the prod if bedding was located only in the escape side. More burrowing was expected to occur when bedding was located in both compartments than when it was located in a single compartment.

\footnotetext{
Method

Subjects. The 24 subjects were naive male Long-Evans rats, weighing 350-450 g, and bred and reared in Trent University's animal colony. They were maintained and housed as in Experiment 1 .
}

Apparatus. The testing apparatus was identical to that used in Experiment 1 with the exception of an aluminum partition used to divide the test chamber into two identical compartments, a prod compartment and an escape compartment. A $12 \times 7.5 \mathrm{~cm}$ opening in the center of the partition at the floor level allowed the animals easy access to both compartments. Latency measures were recorded using a manual keyboard connected to an event recorder.

Procedure. For the prod-side condition, bedding was located only in the prod compartment, whereas for the escape-side condition, it was located only in the escape compartment. For the double-side condition, bedding was located in both compartments. In all three groups, the bedding consisted of $5 \mathrm{~cm}$ of wood shavings. In the prod-side and escape-side conditions, the floor of the empty compartments was lightly sprinkled with wood shavings to make it appear more similar to the compartment with bedding. Eight animals were assigned randomly to each condition.

The initial handling and habituation procedure was the same as in Experiment 1 . In the prod-side and double-side conditions, the prods were located in the same position as in the previous experiment. Since, in the escape-side condition, removal of the bedding from the prod compartment resulted in the prods' being in a higher position relative to the floor than when bedding was present, for half of the animals the prods were adjusted to maintain the same relative position (i.e., $2 \mathrm{~cm}$ above the floor), and for the other half the position of the prods was not adjusted. On Day 5, the rats were placed individually in the center of the escape compartment, were allowed time to enter the prod compartment, and then were shocked, following the same procedure as in Experiment 1. The animals' behavior during the 15 -min period following the shock was videotaped.

From the videotapes, the behaviors analyzed were exploration, immobility, grooming, chewing, burrowing, burying the shock prod, and burying the control prod. Time spent in each behavior, latency to each behavior, and latency to escape (entry into the escape compartment) were recorded. The 2 -sec period immediately following shock was not included because the initial reaction to shock was aiways reflexive withdrawal of the paw, and we were primarily interested in classifying the behavior that occurred after the withdrawal reflex.

\section{Results and Discussion}

Figure 1 shows the mean time spent in each behavior for each of the conditions. A significant main effect of condition was found in mean time spent burrowing $[F(2,21)=4.25, p<.05]$. An orthogonal comparison revealed that the prod-side and escape-side conditions had significantly less burrowing than the

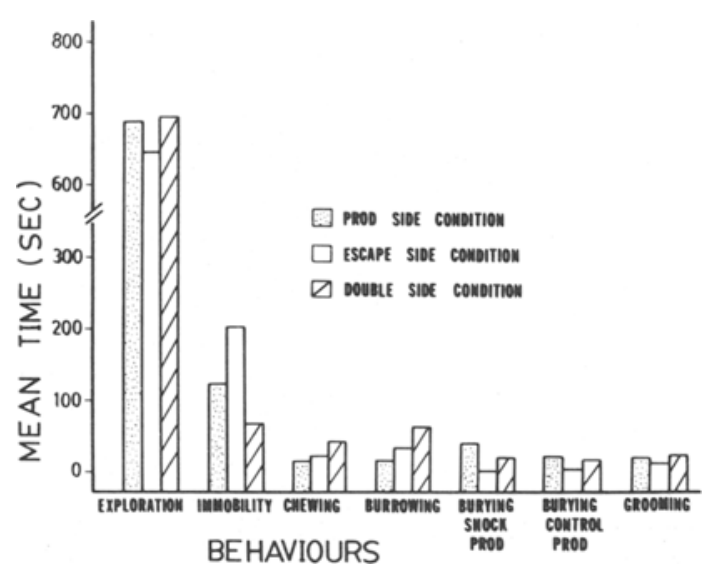

Figure 1. Mean time spent in each behavior in Experiment 2. 
double-side condition $[F(1,21)=6.69, p<.01]$. Significant differences between bedding conditions were also present for time spent burying the shock prod $[\mathrm{F}(2,21)$ $=24.47, p<.001]$ and the control prod $[F(2,21)=$ $8.22, \mathrm{p}<.01$ ]. Burying (both shock and control prods) occurred significantly more in the prod-side and double-side conditions than in the escape-side condition. There were no significant effects of bedding conditions for any of the other behaviors (all ps $>.05$ ).

These results show that burying did not occur when bedding was not readily available and when the animal could escape to a different compartment. In fact, there was not a single occurrence of burying in the escape-side condition, regardless of the position of the prods. Animals made no attempt to transport the bedding from the escape compartment into the prod compartment or to use the small amount of bedding that had either been tracked into the prod compartment or had been sprinkled over the floor. This finding contrasts with Pinel and Treit's (1979) observation that rats would move material from a distance if there was no other material readily available for use in burying. Perhaps the reason for the discrepancy is that, in the Pinel and Treit study, rats did not have the opportunity to escape. However, other differences, such as the use of wood shavings rather than blocks, may also account for the animal's failure to transport the bedding material.

From the summary of the latency data presented in Table 1 , it is evident that burying behavior was not an immediate response to shock. As predicted, burying latencies were longer than latencies to immobility and escape. Although the variance in burying latency was large (ranging from 50-716 sec in animals showing burying), there was not a single case of an animal's burying before engaging in escape and immobility. It should be pointed out that latency to explore also reflects running away from the shock prod because the category of exploration involves a number of locomotive activities. Our finding is at variance with Pinel and Treit's (1979) observation that freezing and fleeing attempts rarely occurred in their studies. McKim and Lett (1979) have also reported relatively long latencies to burying.

The nature of the relationship between burying and burrowing behavior remains uncertain. These two activities involve common behavioral components, but they are differentially affected by the amount and location of bedding. Although there was no significant relationship between the time spent in these behaviors when bedding was located in both compartments $(r=.21)$ or in only the escape compartment $(r=0)$, there was a significant correlation $(r=.93)$ between burrowing and burying when bedding was located only in the prod side. The extent to which these activities are functionally related needs to be investigated further. It is likely that both burying and burrowing are motivated by fear.

In this experiment, time spent burying shock and control prods did not significantly differ for any of the three bedding conditions, although animals in the prod-side condition spent slightly more time burying the shock prod than the control prod $[F(1,7)=4.52$, $\mathrm{p}<.10]$. These results appear contradictory to the finding in Experiment 1 that significantly more time was spent burying the shock prod than the control prod. However, in the previous study, the differences in time spent burying shock and control prods was not significant for each of the two conditions; the difference reached the .05 level of significance only when the two conditions were combined. Furthermore, in both studies animals that did bury typically directed bedding material toward both prods. There were also animals that buried the control prod first and spent more time burying the control than the shock prod. Thus, the overall picture is that most rats did not reliably discriminate between the shock prod and the identical control prod following the single exposure to shock. The present finding is inconsistent with Pinel and Treit's $(1978,1979)$ report that rats selectively buried the shock prod. This discrepancy could be due to differences in strain (see McKim \& Lett, 1979), in the type of prods used, or in extraneous cues present, as well as to differences in the nature or

Table 1

Mean Latencies and Standard Deviations for Each Behavior in Experiment 2

\begin{tabular}{|c|c|c|c|c|c|c|}
\hline & \multicolumn{6}{|c|}{ Condition } \\
\hline & \multicolumn{2}{|c|}{ Prod Side } & \multicolumn{2}{|c|}{ Escape Side } & \multicolumn{2}{|c|}{ Double Side } \\
\hline & Mean & SD & Mean & SD & Mean & SD \\
\hline Exploration & 3.75 & 2.25 & 2.25 & 1.04 & 2.63 & 1.60 \\
\hline Immobility & 3.50 & 2.45 & 9.25 & 10.14 & 9.75 & 9.42 \\
\hline Escape & 15.00 & 16.02 & 10.19 & 14.56 & 17.88 & 15.66 \\
\hline Burrowing & 211.75 & 234.20 & 83.00 & 51.23 & 106.63 & 89.90 \\
\hline Grooming & 251.63 & 286.22 & 253.00 & 169.84 & 154.00 & 114.72 \\
\hline Burying Shock Prod & 287.75 & 270.06 & 900.00 & .00 & 324.00 & 304.13 \\
\hline Burying Control Prod & 512.88 & 315.26 & 900.00 & .00 & 386.75 & 330.02 \\
\hline Chewing & 600.88 & 326.25 & 427.88 & 351.73 & 455.38 & 360.08 \\
\hline
\end{tabular}


intensity of shock. Pinel, Treit, and Wilkie (1980) have identified some of the stimulus attributes involved in prod discrimination. Further research is needed to identify factors that facilitate prod discrimination after a single exposure to shock.

The present results shed some light on the relationship between burying and other defensive behaviors. It appears that there are two stages in avoidance/ escape learning. The initial reactions consist of reflexive behavior (such as leg flexion or paw withdrawal) and Bolles's species-specific defense reactions. These reactions, which are automatic and not readily modifiable by reinforcement, are useful for immediate self-preservation. When the initial automatic reactions fail to remove the organism from immediate danger or from a potentially threatening situation, the second coping stage begins. The second stage involves a broader range of instrumental behavior, which may include burrowing and burying. In terms of the long latencies and lack of consistency, burying behavior appears to belong to the second instrumental stage rather than the initial automatic stage. In other words, burying does not have the characteristics of species-specific defense reactions identified by Bolles (1970).

During the automatic stage, responses incompatible with species-specific defense reactions will be suppressed. However, during the second stage, a variety of instrumental responses may be emitted to cope with the continued presence of aversive stimuli. In the present studies, there are at least two possible explanations as to why burying might emerge as a secondstage behavior. First, burrowing may be high in the response repertoire of the rat and may have a high probability of occurring during the second stage of coping. Burying may be nothing more than a component of this burrowing behavior. There is some evidence to support this view. For example, the mean burrowing latencies were shorter than burying latencies; in the prod-side condition, there was a high correlation between the time spent burrowing and the time spent burying; while burrowing occurred in all animals, only some engaged in burying.

Another interpretive possibility is that, in the second stage, animals sample from their response repertoire and do whatever is necessary to reduce aversive stimulation. A wide variety of behavior will occur. Burying may be an innate instrumental response in an aversive situation; therefore, it is likely to be sam- pled. If burying is effective in removing the threatening stimuli from sight, then it is likely that this activity will increase as a result of negative reinforcement. If burying is not effective in reducing or partially reducing aversive stimulation, then it will be abandoned in favor of other instrumental coping responses. According to this reinforcement hypothesis, burying is more likely to be sampled if this behavior has been reinforced in the past, even in a different context. Pinel et al. (1980) dismiss reinforcement as not being a plausible explanation for burying; however, it appears that such a conclusion is at least premature. Studies need to be designed to assess the role of reinforcement in relation to the burying response.

\section{REFERENCES}

Boice, R. Burrows of wild and albino rats: Effects of domestication, outdoor raising, age, experience, and maternal state. Journal of Comparative and Physiological Psychology, 1977, 91, 649-661.

Bolles, R. C. Species-specific defense reactions and avoidance learning. Psychological Review, 1970, 77, 32-48.

McKıм, W. A., \& LETT, B. T. Spontaneous and shock-induced burying in two strains of rats. Behavioral and Neural Biology, 1979, 26, 76-80.

Pinel, J. P. S., \& TRe IT, D. Burying as a defensive response in rats. Journal of Comparative and Physiological Psychology, 1978, 92, 708-712.

Pinel, J. P. J., \& Treit, D. Conditioned defensive burying in rats: Availability of burying materials. Animal Learning \& Behavior, 1979, 7, 392-396.

Pinel, J. P. J., Treit, D., Ladak, F., \& Maclennan, A. J. Conditioned defensive burying in rats free to escape. Animal Learning \& Behavior, 1980, 8, 447-451.

Pinel, J. P. J., Treit, D., \& Wilkie, D. M. Stimulus control of defensive burying in the rat. Learning and Motivation, 1980, 11, 150-163.

Terlecki, L. J., Pinel, J. P. J., \& Treit, D. Conditioned and unconditioned defensive burying in the rat. Learning and Motivation, 1979, 10, 337-350.

Wilkie, D. M., Maclennan, A. J., \& Pinel, J. P. J. Rat defensive behavior: Burying noxious food. Journal of the Experimental Analysis of Behavior, 1979, 31, 299-306.

Wong, P. T. P. A behavioral field approach to instrumental learning in the rat: I. Partial reinforcement effects and sex differences. Animal Learning \& Behavior, 1977, 5, 5-13.

Wong, P. T. P. A behavioral field approach to general activity: Sex differences and food deprivation in the rat. Animal Learning \& Behavior, 1979, 7, 111-118.

(Manuscript received May 26, 1981 ; revision accepted for publication October 8,1981 .) 